\title{
TRANSTORNO DE SINTOMAS SOMÁTICOS NA POPULAÇÃO RESIDENTE DA ZONA URBANA DE FEIRA DE SANTANA, BAHIA
}

\section{Ludmilla Couto da Silva ${ }^{1}$; Tânia Maria de Araújo ${ }^{2}$ e Técia Maria Santos Carneiro e Cordeiro ${ }^{3}$}

1. Bolsista PIBIC/CNPq, Graduanda de Psicologia, Universidade Estadual de Feira de Santana, e-mail: scmila8@gmail.com

2. Orientadora, Coordenadora do Núcleo de Epidemiologia, Departamento de Saúde, Universidade Estadual de Feira de Santana, e-mail: araujo.tania@uefs.br

3. Pesquisadora do Núcleo de Epidemiologia, Departamento de Saúde, Universidade

Estadual de Feira de Santana, e-mail: teciamarya@yahoo.com.br

PALAVRAS-CHAVE: transtornos mentais; saúde mental; epidemiologia

\section{INTRODUÇÃO}

O transtorno de sintomas somáticos caracteriza-se pela ocorrência de sintomas corporais que não apresentam fundamento patológico ou explicações médicas, mas que produzem sofrimento e prejuízo significativos à saúde mental e física do indivíduo (Coelho, Ávila, 2007; Zorzanelli, 2011). Apesar de comumente não se encontrar uma evidência orgânica para a apresentação do sintoma, não se deve de modo algum, minimizar o sofrimento do indivíduo "somatizador" ou a necessidade de conceder ao mesmo auxílio e cuidados terapêuticos (Fonseca et al., 2008).

A prevalência de transtorno de sintomas somáticos vem crescendo, consideravelmente, especialmente no contexto da atenção primária, transformando-se em um problema de saúde pública a nível mundial (Tófoli, Andrade, Fortes, 2011; Zorzanelli, 2011). As dificuldades no processo de diagnóstico e o encaminhamento terapêutico resultam em frustrações tanto para os profissionais de saúde, quanto para os pacientes, que acabam recebendo um tratamento inadequado, ocasionando a piora do quadro mórbido (Coelho, Ávila, 2007).

O presente estudo teve como objetivo estimar a prevalência de transtorno de sintomas somáticos na população residente da zona urbana de Feira de Santana, Bahia. Esta pesquisa está vinculada ao projeto Caracterização da Saúde Mental de Feira de Santana, Bahia e contempla o objetivo geral deste, que é estimar a prevalência de transtornos mentais em Feira de Santana e seus potenciais fatores de risco associados à sua ocorrência, assim como utiliza as informações populacionais que compõe seu banco de dados.

Caracteriza-se por ser um trabalho de relevância devido à escassez de pesquisas de base populacional que estimem a prevalência desses transtornos e a necessidade de uma melhor compreensão e reconhecimento dessa condição por parte dos profissionais de saúde e das autoridades governamentais. Os resultados poderão contribuir para uma melhor reflexão sobre o tratamento do transtorno de sintomas somáticos e transtornos relacionados, viabilizando o planejamento de projetos de intervenção que viabilizem uma abordagem mais adequada visando a recuperação do paciente e a viabilidade de um acompanhamento psicológico e psiquiátrico.

\section{METODOLOGIA}

Este estudo é um recorte da Pesquisa 'Caracterização da Saúde Mental de Feira de Santana, Bahia, Brasil' desenvolvido pelo Núcleo de Epidemiologia (NEPI) da Universidade Estadual de Feira de Santana (UEFS). Trata-se de um estudo 
epidemiológico de corte transversal incluindo amostra representativa da população residente da zona urbana de Feira de Santana, Bahia, Brasil.

Neste estudo foram estudados os residentes da zona urbana de Feira de Santana, Bahia. Os dados foram coletados mediante a aplicação de uma ficha domiciliar e um questionário estruturado composto por blocos de questões. A variável desfecho foi o transtorno de sintomas somáticos e para mensurá-la foi utilizado o Patient Health Questionnaire (PHQ) (SANTOS et al, 2013). Para a análise dos efeitos sobre a variável desfecho referida foram empregadas como variáveis de exposição: características sociodemográficas, ocupacionais, atos de violência, doenças auto-referidas e hábitos de vida.

As técnicas envolvidas foram a análise bivariada e multivariada. Para análise das variáveis de interesse, foi utilizado o programa estatístico "Statistical Package for the Social Sciences" (SPSS) for Windows, versão 15.0. e OpenEpi versão 3.03. Foram calculadas as medidas de associação (razão de prevalência) e seus respectivos Intervalos de Confiança de 95\%, assumindo-se, para significância estatística, $\mathrm{p} \leq 0,05$. Foi feita uma pré-seleção das variáveis que entrariam no Modelo de Regressão Logística, tendo como critério de seleção valor de $p \leq 0,25$ tendo significância estatística.

Os protocolos de pesquisa, ao qual este estudo integra, foram avaliados e aprovados pelo Comitê da Ética em Pesquisa com Seres Humanos da Universidade Estadual de Feira de Santana sob parecer n042/06.

\section{RESULTADOS E DISCUSSÕES}

Foram estudados 4.167 residentes da zona urbana de Feira de Santana, Bahia. No que diz respeito às características sociodemográficas, observou-se maior percentual de trabalhadoras $(67,6 \%)$, entre a faixa etária de 30 anos ou menos (40,5\%), sem companheiro $(51,4 \%)$, sem ensino superior $(97,9 \%)$ e que autorreferiram cor de pele não branca $(84,5 \%)$. Com relação à renda mensal, a maior parte dos indivíduos recebia até um salário mínimo $(52,6 \%)$, possuía vínculo de trabalho informal $(51,3 \%)$ e não exercia outra atividade que remunerada $(86,4 \%)$.

No que se refere aos hábitos de vida, houve predomínio de indivíduos praticantes de atividades de lazer $(86,5 \%)$ e de não praticantes de atividades físicas $(61,8 \%)$. Quanto ao consumo de álcool, a maior proporção da população estudada não consumia bebida alcoólica $(71,3 \%)$. A maior parte dos indivíduos era de não fumantes $(88,3 \%)$.

Em relação à ocorrência de atos de violência nos últimos 24 meses, a maioria dos indivíduos não havia sofrido agressão física ou psicológica $(81,4 \%)$, assim como não havia sido vítima de acidente de trânsito $(95,2 \%)$. No que concerne à ocorrência de atos violentos na infância, a maior parte da população estudada relatou não ter sofrido agressão física quando criança (88\%).

Acerca das vivências do último mês, constatou-se que a maior proporção dos indivíduos estudados relatou não ter se incomodado com algo ruim que havia acontecido recentemente (64\%), bem como a preocupação com a saúde $(52,3 \%)$ não havia sido um incômodo para os mesmos.

A prevalência de transtorno de sintomas somáticos entre os residentes da zona urbana de Feira de Santana, Bahia, foi de $8,6 \%$, sendo que todos estes indivíduos eram do sexo feminino (12,7\%). Segundo Sadock et al. (2007) cerca de 0,2 a 2,0\% das mulheres são acometidas por transtornos de sintomas somáticos durante toda a vida e no que se refere ás distinções de manifestação do mesmo segundo gênero, o desenvolvimento do mesmo em mulheres é de 5 a 20 vezes mais provável do que em homens. 
Observou-se associações positivas estaticamente significantes para: idade de 31 a 45 anos $(\mathrm{RP}=1,47$; valor de $\mathrm{p}<0,01)$, receber até 1 salário mínimo $(\mathrm{RP}=1,77$; valor de $\mathrm{p}<0,01)$, ter vínculo de trabalho informal $(\mathrm{RP}=1,63$; valor de $\mathrm{p}=0,01)$, estar desempregado $(\mathrm{RP}=2,01$; valor de $\mathrm{p}<0,01)$, praticar atividade de lazer $(\mathrm{RP}=1,46$; valor de $\mathrm{p}<0,01)$, realizar atividade física $(\mathrm{RP}=1,75$; valor de $\mathrm{p}<0,01)$, ser fumante $(\mathrm{RP}=1,45$; valor de $\mathrm{p}<0,01)$, já ter sofrido agressão psicológica e física $(R P=2,33$; valor de $\mathrm{p}<0,01)$; ter se incomodado pouco com relação à preocupação com a saúde $(\mathrm{RP}=1,64$; valor de $\mathrm{p}<0,01)$, ter se incomodado muito com relação à preocupação com a saúde $(\mathrm{RP}=4,02$; valor de $\mathrm{p}<0,01)$ e ter se incomodado muito com algo ruim que aconteceu recentemente $(\mathrm{RP}=3,56$; valor de $\mathrm{p}<0,01)$. Enquanto que a associação negativa foi estaticamente significante apenas para idade de mais de 45 anos $(\mathrm{RP}=0,27$; valor de $\mathrm{p}<0,01)$, ter companheiro $(\mathrm{RP}=0,37$; valor de $\mathrm{p}<0,01)$ e ter cor de pele branca $(\mathrm{RP}=0,57$, valor de $\mathrm{p}<0,01)$.

Após análise multivariada, utilizando regressão logística, as variáveis que permaneceram associadas aos transtornos de sintomas somáticos foram: ter idade de 31 a 45 anos $(R P=0,93$; valor de $\mathrm{p}=0,64)$, ter mais de 45 anos de idade $(R P=0,96$; valor de $\mathrm{p}<0,01)$, ter companheiro $(\mathrm{RP}=0,33$; valor de $\mathrm{p}<0,01)$, ter até 1 salário mínimo $(\mathrm{RP}=$ $1,58$; valor de $\mathrm{p}<0,01)$, realizar atividade física $(\mathrm{RP}=1,68$; valor de $\mathrm{p}<0,01)$, ter sofrido agressão psicológica ou física $(\mathrm{RP}=1,52$; valor de $\mathrm{p}=0,01)$, ter sido vítima de agressão na infância $(\mathrm{RP}=1,74$; valor de $\mathrm{p}<0,01)$, ter se incomodado pouco com relação à preocupação com a saúde ( $\mathrm{RP}=1,70$; valor de $\mathrm{p}=0,01)$, ter se incomodado muito com relação à preocupação com a saúde $(\mathrm{RP}=2,73$; valor de $\mathrm{p}<0,01)$, ter se incomodado pouco com algo ruim que aconteceu recentemente $(\mathrm{RP}=1,58$; valor de $\mathrm{p}=0,07)$ e ter se incomodado muito com algo ruim que aconteceu recentemente $(\mathrm{RP}=1,92$; valor de $\mathrm{p}<0,01)$.

A baixa remuneração está relacionada ao desencadeamento de diversos problemas sociais, como a desqualificação da ocupação que se exerce e a condições de vida instáveis, que repercutem negativamente na qualidade de vida, podendo, provocar, por conseguinte, adoecimento psíquico (Souza et al., 2017). Segundo Carvalho (2010) as dificuldades laborais ou econômicas podem constituir fatores que tornam o indivíduo suscetível ao desenvolvimento de transtorno de sintomas somáticos.

A vivência de agressões físicas, sexuais ou emocionais na infância de mulheres pode prejudicar o adequado desenvolvimento biológico e psicológico da criança (Felitti et al., 1998), o que significa que os efeitos da violência podem se suceder em curto, médio e longos prazos (Silva, Ferraz, 2009). Andrade et al. (2006) afirma que o desenvolvimento de alguns transtornos, dentre os quais os transtornos de sintomas somáticos, ocorre como consequiência de algum tipo de violência sofrida.

Os atos de violência são apontados como experiências potencialmente adoecedoras, podendo repercutir na saúde física, mental e reprodutiva da mulher (Andrade et al., 2006). Day et al. (2003) afirma que frequentemente as consequiências psicológicas do abuso emergem de modo ainda mais intenso que suas repercussões físicas. A vivência do abuso incide negativamente sobre a autoestima da mulher, sujeitando as mesmas a um risco maior de desenvolverem morbidades psíquicas, como o estresse pós-traumático, depressão e o uso abusivo de álcool e drogas.

\section{CONSIDERAÇÕES FINAIS}

Sabe-se que as questões de gênero ainda constituem o fundamento pelo qual as relações sociais e de trabalho são construídas. As mulheres, as únicas acometidas por transtornos de sintomas somáticos neste estudo, apesar de vivenciarem, na última década, avanços consideráveis no que diz respeito à igualdade de direitos nos distintos 
contextos da sociedade, ainda sofrem intensa vulnerabilidade no que se refere aos atos de violência, os quais podem ocorrer a qualquer momento da vida das mesmas.

Outro aspecto a ser destacado é a associação entre receber até um salário mínimo e ter suspeita diagnóstica do referido transtorno, que revela como as questões de gênero ainda reverberam nas condições de permanência, nas relações e na organização laboral, em que as mulheres estão sujeitas a desigualdade na remuneração, na qualidade do emprego e no acesso a benefícios e direitos sociais quando comparado aos homens. Este contexto de precarização laboral vivenciado pelas mulheres constitui terreno fértil para o adoecimento mental, visto que ele gera instabilidade, insatisfação, angústia e sofrimento psíquico.

Diante desses dados, é possível refletir e planejar projetos de prevenção, assim como de intervenção e sistematização de políticas públicas que proteja e promova a saúde dessas mulheres, assim como previna os homens do adoecimento.

\section{REFERÊNCIAS}

ANDRADE, L. H. S. G.; VIANA, M. C.; SILVEIRA, C. M. 2006. Epidemiologia dos transtornos psiquiátricos na mulher. Rev. psiquiatr. clín., 33 (2): 43-54.

CARVALHO, J.G. 2010. Transtornos somatoformes na atenção básica à saúde - uma revisão de literatura. Universidade Federal de Minas Gerais, Tese.

COELHO, C.L.S.; ÁVILA, L.A. 2007. Controvérsias sobre a somatização. Rev. Psiq. Clin, 34 (6): 278-284.

DAY, V. P.; TELlES, L. E. B.; ZORATTO, P. H.; AZAMBUJA, M. R. F.; MACHADO, D. A.; SILVEIRA, M. B; DEBIAGGI, M.; REIS, M. G. CARDOSO, R. G.; BLANK, P. 2003. Violência doméstica e suas diferentes manifestações. $R$. Psiquiatr. RS, 25 (1): 9-21.

FELITTI, V. J.; ANDA, R. F.; NORDENBERG, D.; WILLIAMSON, D. F.; EDWARDS, V.; KOSS, M. P.; MARKS, J. S. 1998. Relationship of childhood abuse and household dysfunction to many of the leading causes of death in adults: The Adverse Childhood Experiences (ACE) study. Am J Prev Med, 14 (4): 245-58.

SADOCK, B. J.; KAPLAN, H. I.; SADOCK, V. A. 2007. Compêndio de Psiquiatria: ciências do comportamento e psiquiatria clinica. Porto Alegre, Artmed, 1584p.

SILVA, C. P.; FERRAZ, R. R. N. 2009. Estudo da prevalência de transtornos psíquicos associados à violência em usuários atendidos no Serviço de Psicologia do Município de Embu Guaçu - SP. ConScientiae Saúde, 8, (1): 105-110.

SOUZA, L. P. S.; BARBOSA, B. B.; SILVA, C. S. O.; SOUZA, A. G.; FERREIRA, T. N.; SIQUEIRA, L. G. 2017. Prevalência de transtornos mentais comuns em adultos no contexto da Atenção Primária à Saúde. Revista Portuguesa de Enfermagem de Saúde Mental, (18): 59-66.

TOFOLI, L. F.; ANDRADE, L. H.; FORTES, S. 2011. Somatização na América Latina: uma revisão sobre a classificação de transtornos somatoformes, síndromes funcionais e sintomas sem explicação médica. Rev. Bras. Psiquiatr., 33 (1): 59-69. 